PROCEEDINGS OF THE

AMERICAN MATHEMATICAL SOCIETY

Volume 129, Number 12, Pages 3715-3719

S 0002-9939(01)05989-5

Article electronically published on July 10, 2001

\title{
A UNIVERSAL COANALYTIC LINEAR ORDERING
}

\author{
ABHIJIT DASGUPTA \\ (Communicated by Carl G. Jockusch, Jr.)
}

\begin{abstract}
We construct a $\Pi_{1}^{1}$ linear ordering in which every $\boldsymbol{\Pi}_{1}^{\mathbf{1}}$ (coanalytic) linear ordering can be order embedded.
\end{abstract}

\section{INTRODUCTION}

We say that $\left(A,<_{A}\right)$ is a Borel (resp. coanalytic) linear ordering if $A$ is a set of reals, $<_{A}$ is a linear (total) ordering of $A$, and both $A$ and $<_{A}$ are Borel (resp. coanalytic).

By the structure theorem on Borel orderings in [2], given any Borel linear ordering $\left(A,<_{A}\right)$, there is a Borel linear ordering $\left(B,<_{B}\right)$ such that $\left(A,<_{A}\right)$ can be order embedded in $\left(B,<_{B}\right)$, but $\left(B,<_{B}\right)$ cannot be order embedded in $\left(A,<_{A}\right)$. It follows that there is no universal Borel linear ordering with respect to order embeddability.

In this paper we show that among coanalytic linear orderings, on the other hand, there is a universal one:

Theorem 1.1. There exists a $\Pi_{1}^{1}$ set $P$ of reals and $a \Pi_{1}^{1}$ relation $<_{P} \subseteq P \times P$ which linearly orders $P$ and satisfies the following: if $A$ is a set of reals and $<_{A} \subseteq A \times A$ is any $\mathbf{\Pi}_{\mathbf{1}}^{\mathbf{1}}$ relation which linearly orders $A$, then $\left(A,<_{A}\right)$ is order embeddable in $\left(P,<_{P}\right)$.

\section{Preliminaries}

Unless otherwise noted, we use notation, terminology, and results of modern descriptive set theory as can be found in a standard textbook like [3], 4], or [6]. The facts about linear orderings used below can be found in [5]; here we review those which we will need. We also use results from [1] and [2] about Borel orderings.

We use the standard notation of set theory for ordinals: $\omega$, the smallest infinite (countable) ordinal, is the set of all natural numbers (finite ordinals); and $\omega_{1}$, the least uncountable ordinal, is the set of all countable ordinals.

A linear ordering is an ordered pair $\left(A,<_{A}\right)$ where $A$ is a set and $<_{A} \subseteq A \times A$ is a relation on $A$ such that $<_{A}$ is transitive and satisfies the rule of trichotomy on $A$ : For any $x, y \in A$, exactly one of the conditions $x=y, x<_{A} y$, and $y<_{A} x$ holds. In this case we say that $<_{A}$ linearly orders $A$.

Received by the editors November 22, 1999 and, in revised form, May 1, 2000.

2000 Mathematics Subject Classification. Primary 03E15, 04A15; Secondary 06A05.

Key words and phrases. Descriptive set theory, coanalytic sets, total order, linear order.

(C)2001 Abhijit Dasgupta, GNU GPL style copyleft 3715 
Given linear orderings $\left(A,<_{A}\right),\left(B,<_{B}\right)$ and a function $f: A \rightarrow B$, we say that $f$ is (strictly) increasing or order preserving if for all $x, y \in A$, if $x<_{A} y$, then $f(x)<_{B} f(y)$. We say that $\left(A,<_{A}\right)$ is embeddable in $\left(B,<_{B}\right)$ if there is an order preserving $f: A \rightarrow B$.

If $\left(A,<_{A}\right)$ is a linear ordering and $B, C \subseteq A$, we write $B<_{A} C$ to mean $x<_{A} y$ for all $x \in B$ and $y \in C$.

A linear ordering is short if it does not contain any increasing or decreasing $\omega_{1}$-chain.

If $\xi$ is an ordinal and $\left(A,<_{A}\right)$ is a linear ordering, then we define the $\xi$-fold lexicographic power of $\left(A,<_{A}\right)$ as the ordering on the set $A^{\xi}$ (of all $\xi$-sequences of members of $A$ ) where for $x, y \in A^{\xi}, x$ precedes $y$ iff there exists $\alpha<\xi$ such that $x(\alpha)<_{A} y(\alpha)$ and for all $\beta<\alpha, x(\beta)=y(\beta)$; it is easily verified that this indeed defines a linear ordering. In particular, $2=\{0,1\}$ is ordered naturally, and $2^{\xi}$ denotes its $\xi$-fold lexicographic power. Let $2^{\triangleleft \omega_{1}}$ denote the subordering of $2^{\omega_{1}}$ consisting of those elements $x$ of $2^{\omega_{1}}$ for which there is $\zeta<\omega_{1}$ with $x(\zeta)=1$ and $x(\beta)=0$ for all $\beta$ with $\zeta<\beta<\omega_{1}$ (i.e. binary $\omega_{1}$-sequences with a last 1 ). $\left(\omega^{\omega},<\mathcal{N}\right)$ denotes the $\omega$-fold lexicographic power of $(\omega,<)$.

A linear ordering $\left(A,<_{A}\right)$ is an $\eta_{1}$-ordering if for any countable subsets $B, C \subseteq A$ with $B<_{A} C$, there exists $x \in A$ with $B<_{A}\{x\}<_{A} C$.

A linear ordering $\left(A,<_{A}\right)$ is quasi-short if there are subsets $A_{\alpha} \subseteq A$ for $\alpha<\omega_{1}$ such that $A=\bigcup\left\{A_{\alpha} \mid \alpha<\omega_{1}\right\}$, and for each $\alpha<\omega_{1}$, the restriction $<_{A} \mid A_{\alpha}$ of $<_{A}$ to $A_{\alpha}$ is short.

Lemma 2.1. 1. A subordering of a short linear ordering is short.

2. The union of countably many short linear orderings is short. More precisely, if $\left(A,<_{A}\right)$ is a linear ordering with $A=\bigcup\left\{A_{n} \mid n \in \omega\right\}$ and $\left(A_{n},<_{A}\left\lceil A_{n}\right)\right.$ is short for each $n$, then $\left(A,<_{A}\right)$ is short.

3. Suppose that $\left(A,<_{A}\right)$ is a linear ordering, $B \subseteq A$ is a short subordering, and whenever $x, y, z \in A$ and $x<_{A} y<_{A} z$, there is $w \in B$ with $x<_{A} w<_{A} z$. Then $\left(A,<_{A}\right)$ is short.

4. If $\xi$ is a countable ordinal, then the $\xi$-fold lexicographic power of a short linear ordering is short. In particular, $2^{\xi}$ is short for countable $\xi$.

5. $2^{\triangleleft \omega_{1}}$ is an $\eta_{1}$-ordering.

Proof. The first three statements are obvious, and the fourth is verified by induction on $\xi$ using the second and third claims. The last statement is an immediate consequence of Theorem 9.24 on page 165 of [5].

\section{EMBEDDING QUASI-SHORT LINEAR ORDERINGS INTO $\eta_{1}$-ORDERINGS}

Theorem 3.1. Every quasi-short linear ordering can be embedded into any $\eta_{1}$ ordering.

Proof. This follows from the following lemma.

Lemma 3.2 (Hausdorff). If $\left(A,<_{A}\right)$ is a short linear ordering, $\left(B,<_{B}\right)$ is an $\eta_{1}$ ordering, $S \subseteq A$, and $f: S \rightarrow B$ is strictly increasing, then $f$ can be extended to a strictly increasing map from all of $A$ into $B$.

Proof. Let $\mathcal{F}$ be the family of all strictly increasing functions extending $f$ (mapping a subset of $A$ into $B$ ). Partially order $\mathcal{F}$ under extension. Then by Zorn's lemma, there is a maximal member $g$ of $\mathcal{F}$. We claim that $\operatorname{dom}(g)=A$. Otherwise there 
would exist $a \in A \backslash \operatorname{dom}(g)$. Let $L=\left\{x \in \operatorname{dom}(g) \mid x<_{A} a\right\}$ and $R=\{x \in \operatorname{dom}(g) \mid$ $\left.a<_{A} x\right\}$. Since $\left(A,<_{A}\right)$ is short, there exist countable sets $P$ and $Q$ with $P \subseteq L$ and $Q \subseteq R$ such that: (a) for all $x \in L$, there is $y \in P$ with $x<_{A} y$ or $x=y$; and (b) for all $x \in R$, there is $y \in Q$ with $y<_{A} x$ or $x=y$. Put $C=\{g(x) \mid x \in P\}$ and $D=\{g(x) \mid x \in Q\}$. Then $C$ and $D$ are countable subsets of $B$ with $C<_{B} D$. Since $\left(B,<_{B}\right)$ is an $\eta_{1}$-ordering, there is $b \in B$ with $C<_{B}\{b\}<_{B} D$. Define an extension $h$ of $g$ with $\operatorname{dom}(h)=\operatorname{dom}(g) \cup\{a\}$ by setting $h(a)=b$. Then $h$ is a strictly increasing proper extension of $g$, contradicting the maximality of $g$.

To finish the proof of the theorem, let $\left(A,<_{A}\right)$ be a quasi-short linear ordering, and let $A_{\alpha} \subseteq A$ for $\alpha<\omega_{1}$ be such that $A=\bigcup\left\{A_{\alpha} \mid \alpha<\omega_{1}\right\}$, and for each $\alpha<\omega_{1}$, the restriction $<_{A}\left\lceil A_{\alpha}\right.$ of $<_{A}$ to $A_{\alpha}$ is short. Without loss of generality, we assume that the sets $A_{\alpha}$ increase with $\alpha$. Now let $\left(B,<_{B}\right)$ be any $\eta_{1}$-ordering. Using Lemma 3.2 and transfinite induction, we can build strictly increasing functions $f_{\alpha}: A_{\alpha} \rightarrow B, \alpha<\omega_{1}$, such that if $\alpha<\beta<\omega_{1}$, then $f_{\beta}$ extends $f_{\alpha}$. Then the common extension of all the functions $f_{\alpha}$ is a strictly increasing map from $\left(A,<_{A}\right)$ to $\left(B,<_{B}\right)$.

\section{CoAnalytic ORDERINGS ARE QUASI-SHORT}

We will use the fact that Borel linear orderings are short ([1], [2]) in the proof of the following theorem.

Theorem 4.1. Let $A$ be a set of reals and $<_{A}$ be a linear ordering of $A$ such that $<_{A}$, as a subset of the plane, is $\mathbf{\Pi}_{\mathbf{1}}^{1}$ (coanalytic). Then $\left(A,<_{A}\right)$ is quasi-short.

Proof. We assume that $A$ is uncountable. $A$ is $\boldsymbol{\Sigma}_{\mathbf{2}}^{\mathbf{1}}$, since $<_{A}$ is $\boldsymbol{\Pi}_{\mathbf{1}}^{\mathbf{1}}$ and $x \in A$ iff $\exists y\left(x<_{A} y \vee y<_{A} x\right)$. So we can express $A$ as a union of $\omega_{1}$ Borel sets:

$$
A=\bigcup_{\xi<\omega_{1}} A_{\xi}
$$

where each $A_{\xi}$ is a Borel set. For each $\xi<\omega_{1}$, let

$$
\begin{array}{lll}
<_{\xi} & \stackrel{\text { def }}{=} & \left\{(x, y) \in A_{\xi} \times A_{\xi} \mid x<_{A} y\right\}, \\
>_{\xi} \quad \stackrel{\text { def }}{=} & \left\{(x, y) \in A_{\xi} \times A_{\xi} \mid y<_{A} x\right\}, \\
=_{\xi} \stackrel{\text { def }}{=} & \left\{(x, y) \in A_{\xi} \times A_{\xi} \mid x=y\right\} .
\end{array}
$$

Since linear orderings satisfy the rule of trichotomy, the three $\Pi_{1}^{1}$ sets $<_{\xi},>_{\xi}$, and $={ }_{\xi}$ form a partition of the Borel set $A_{\xi} \times A_{\xi}$. So by Suslin's theorem, $<_{\xi},>_{\xi}$, and $=\xi$ are all Borel. So $\left(A_{\xi},<_{\xi}\right)$ is a Borel linear ordering, and therefore short, for each $\xi<\omega_{1}$. Hence $\left(A,<_{A}\right)$ is quasi-short.

\section{A $\Pi_{1}^{1}$ ORDERING CONTAINING AN $\eta_{1}$-ORDERING}

Theorem 5.1. There exist $a \Pi_{1}^{1}$ set $P$ of reals and $a \Pi_{1}^{1}$ linear ordering $<_{P}$ of $P$ such that $2^{\triangleleft \omega_{1}}$ can be embedded in $\left(P,<_{P}\right)$.

Proof. Throughout this proof we use terminology and results from [4]. We work in the space of irrationals $\mathcal{N}=\omega^{\omega}$. For $i, j \in \omega$, let $\langle i, j\rangle \in \omega$ be defined as $\langle i, j\rangle=2^{i}(2 j+1)-1$. For $x \in \omega^{\omega}$, and $i \in \omega$, define $(x)_{i} \in \omega^{\omega}$ by

$$
(x)_{i}(n)=x(\langle i, n\rangle)(n \in \omega) .
$$


For $x \in \omega^{\omega}$ and $n \in \omega$, let

$$
\begin{array}{rll}
\leq_{x} & \stackrel{\text { def }}{=} & \{(i, j) \mid x(\langle i, j\rangle)=1\}, \\
<_{x} & \stackrel{\text { def }}{=} & \left\{(i, j) \mid i \leq_{x} j \wedge i \neq j\right\}, \\
\Phi[x] & \stackrel{\text { def }}{=} & \text { the field of } \leq_{x} \\
& = & \left\{i \mid \exists j\left(i \leq_{x} j \vee j \leq_{x} i\right)\right\}, \\
\Omega[x] & \stackrel{\text { def }}{=} & \text { the subset of } \omega \text { coded by } x \\
= & \{i \mid x(i)=1\}, \\
<_{x}[n] & \stackrel{\text { def }}{=} & \text { the }<_{x} \text {-predecessors of } n \\
& =\left\{i \mid i<_{x} n\right\} .
\end{array}
$$

The following relations on $\omega^{\omega}$ are arithmetical:

$$
\begin{aligned}
& \mathrm{LO}(x) \stackrel{\text { def }}{\longleftrightarrow \quad} \quad x \text { codes a linear ordering } \\
&(\forall n \in \Phi[x])\left(n \leq_{x} n\right) \\
& \wedge(\forall m, n)\left(\left(m \leq_{x} n \wedge n \leq_{x} m\right) \longrightarrow m=n\right) \\
& \wedge(\forall m, n, k)\left(\left(m \leq_{x} n \wedge n \leq_{x} k\right) \longrightarrow m \leq_{x} k\right) \\
& \wedge(\forall m, n \in \Phi[x])\left(m \leq_{x} n \vee n \leq_{x} m\right), \\
& \mathrm{ISO}(f, x, y) \stackrel{\mathrm{def}}{\longleftrightarrow \quad} \quad \mathrm{O}(x) \text { and } \mathrm{LO}(y) \text { and (either }(f \uparrow \Phi[x] \text { maps } \Phi[x] \\
& \text { bijectively onto an initial segment of }<_{y} \text { preserving } \\
&\text { order from } \left.<_{x} \text { to }<_{y} \text { and } f=0 \text { outside } \Phi[x]\right) \text { or } \\
&(\exists n \in \Phi[x])\left(f \left\lceil<_{x}[n] \text { maps }<_{x}[n]\right.\right. \text { bijectively } \\
& \text { onto } \Phi[y] \text { preserving order from }<_{x} \text { to }<_{y} \text { and } f=0 \\
&\text { outside } \left.\left.<_{x}[n]\right)\right) .
\end{aligned}
$$

So if $\mathrm{LO}(x)$, then $<_{x}$ is a linear ordering of a subset of $\omega$. If $\mathrm{LO}(x)$ and $n \in \omega$, then let $|x|$ denote the order-type of $<_{x}$, and $|x|_{\prec n}$ the order-type of $<_{x}[n]$. Let

$$
\mathrm{WO}(x) \stackrel{\text { def }}{\longleftrightarrow} \mathrm{LO}(x) \text { and }<_{x} \text { is a well-ordering. }
$$

Then WO is $\Pi_{1}^{1}$, and so are the relations " $|x|=|y|$ " and " $|x|<|y|$ " defined on WO. Note that

$$
\mathrm{WO}(x) \wedge \mathrm{WO}(y) \longrightarrow(\exists ! f)(\mathrm{ISO}(f, x, y)) .
$$

We now define the $\Pi_{1}^{1}$ set $P$ by

$$
\begin{aligned}
P(x) \stackrel{\text { def }}{\longleftrightarrow} & (x)_{0} \text { codes a successor ordinal and }(x)_{1} \text { codes } \\
& \text { a subset of } \Phi\left[(x)_{0}\right] \text { "containing" sup }\left|(x)_{0}\right| \\
& \mathrm{WO}\left((x)_{0}\right) \wedge \Omega\left[(x)_{1}\right] \subseteq \Phi\left[(x)_{0}\right] \\
& \wedge\left(\exists n \in \Phi\left[(x)_{0}\right]\right)\left(n \in \Omega\left[(x)_{1}\right] \wedge\left(\forall i \in \Phi\left[(x)_{0}\right]\right)\left(i \leq_{x} n\right)\right) .
\end{aligned}
$$

Notice that $P$ codes $2^{\triangleleft \omega_{1}}$, i.e. there is a natural surjection from $P$ onto $2^{\triangleleft \omega_{1}}$ : If $x \in P$, then $(x)_{0}$ codes a countable successor ordinal $\left|(x)_{0}\right|$ with greatest element $\sup \left|(x)_{0}\right|$, and $(x)_{1}$ codes a subset of $\left|(x)_{0}\right|$ containing sup $\left|(x)_{0}\right|$. So for $x \in P$, we can define $a_{x} \in 2^{\omega_{1}}$ by

$$
a_{x}(\zeta)= \begin{cases}1 & \text { if }(\exists n)\left(\zeta=\left|(x)_{0}\right|_{\prec n} \wedge(x)_{1}(n)=1\right), \quad\left(\zeta<\omega_{1}\right) \\ 0 & \text { otherwise }\end{cases}
$$


Define a linear ordering $<_{P}$ on $P$ by

$$
x<_{P} y \stackrel{\text { def }}{\longleftrightarrow} a_{x}<a_{y} \text { in } 2^{\triangleleft \omega_{1}} \vee\left(a_{x}=a_{y} \wedge x<\mathcal{N} y\right) .
$$

Recall that $<_{\mathcal{N}}$ is the $\omega$-fold lexicographic power of $(\omega,<)$ (here, in place of $<_{\mathcal{N}}$, any arithmetical linear ordering of $\omega^{\omega}$ will do).

Note that by definition of $<_{P}, 2^{\triangleleft \omega_{1}}$ can be embedded in $\left(P,<_{P}\right)$.

It remains to show that $<_{P}$ is $\Pi_{1}^{1}$. First:

$$
\begin{aligned}
a_{x}<a_{y} \longleftrightarrow & P(x) \wedge P(y) \wedge(\forall f)\left(\operatorname{ISO}\left(f,(x)_{0},(y)_{0}\right)\right. \\
& \longrightarrow\left[( \exists n \in \Phi [ ( x ) _ { 0 } ] ) \left((x)_{1}(n) \neq 1 \wedge(y)_{1}(f(n))=1 \wedge\right.\right. \\
& \left.\left.\left(\forall i \in \Phi\left[(x)_{0}\right]\right)\left(i<_{x} n \longrightarrow\left((x)_{1}(i)=1 \leftrightarrow(y)_{1}(f(i))=1\right)\right)\right)\right] \\
& \vee\left[\left(\forall i \in \Phi\left[(x)_{0}\right]\right)\left((x)_{1}(i)=1 \leftrightarrow(y)_{1}(f(i))=1\right)\right. \\
& \left.\left.\wedge\left|(x)_{0}\right|<\left|(y)_{0}\right|\right]\right) .
\end{aligned}
$$

Next,

$$
\begin{aligned}
a_{x}=a_{y} \longleftrightarrow & P(x) \wedge P(y) \wedge\left|(x)_{0}\right|=\left|(y)_{0}\right| \wedge(\forall f)\left(\operatorname{ISO}\left(f,(x)_{0},(y)_{0}\right)\right. \\
& \left.\longrightarrow\left(\forall i \in \Phi\left[(x)_{0}\right]\right)\left((x)_{1}(i)=1 \leftrightarrow(y)_{1}(f(i))=1\right)\right) .
\end{aligned}
$$

Finally, $<_{\mathcal{N}}$ is an arithmetical linear ordering of $\omega^{\omega}$.

It follows that $<_{P}$ is $\Pi_{1}^{1}$.

Theorem 1.1 is now immediate from Theorem 4.1, Theorem 3.1 and Theorem 5.1.

\section{REMARKS}

Let us call a linear ordering very short if it can be embedded in $2^{\alpha}$ for some countable ordinal $\alpha$. Thus very short linear orderings are short.

Harrington and Shelah in [1] first proved that Borel linear orderings are short. Harrington, Marker, and Shelah in [2] show that Borel linear orderings are very short. Since for each countable ordinal $\alpha$ there is a Borel linear ordering order isomorphic to $2^{\alpha}$, it also follows that there is no universal Borel linear ordering. Note that the authors in [2] studied pre-orderings (i.e. reflexive transitive relations), whereas here we looked only at (strict) linear orderings (i.e. relations which are asymmetric, transitive, and satisfy the rule of trichotomy).

On the other hand, there are short coanalytic linear orderings which are not very short. To see this, define an ordering $<_{S}$ on $P$ :

$$
x<_{S} y \stackrel{\text { def }}{\longleftrightarrow} P(x) \wedge P(y) \wedge\left((x)_{0}<\mathcal{N}(y)_{0} \vee\left((x)_{0}=(y)_{0} \wedge a_{x}<a_{y}\right)\right) .
$$

\section{REFERENCES}

1. L. Harrington and S. Shelah, Counting equivalence classes for co- $\kappa$-Souslin relations, Logic Colloquium 1980, Eds. D. Van Dalen, D. Lascar, and T. J. Smiley, North-Holland, 1982. MR 84c:03088

2. L. Harrington, D. Marker, and S. Shelah, Borel Orderings, Trans. Amer. Math. Soc. 310, 1 (1988), 293-302. MR 90c:03041

3. A. S. Kechris, Classical Descriptive Set Theory, Springer-Verlag, 1994.

4. Y. N. Moschovakis, Descriptive Set Theory, North-Holland, 1980. MR 82e:03002

5. J. G. Rosenstein, Linear Orderings, Academic Press, 1982. MR 84m:06001

6. S. M. Srivastava, A Course on Borel Sets, Springer-Verlag, 1998. MR 99d:04002

E-mail address: takdoom@yahoo.com 